\title{
Experimental determination of the quadratic nonlinear magnetic susceptibility of a varactor-loaded split ring resonator metamaterial
}

\author{
Stéphane Larouche, ${ }^{\text {a) }}$ Alec Rose, Ekaterina Poutrina, Da Huang, and David R. Smith \\ Department of Electrical and Computer Engineering, Center for Metamaterials and Integrated Plasmonics, \\ Pratt School of Engineering, Duke University, Box 90291, Durham, North Carolina 27708, USA
}

(Received 28 April 2010; accepted 15 June 2010; published online 9 July 2010)

\begin{abstract}
This letter presents a quantitative measurement of the second harmonic generated by a slab of varactor loaded split ring resonator metamaterial and the retrieval of the effective quadratic nonlinear magnetic susceptibility $\chi_{\mathrm{m}}^{(2)}$ using an approach based on transfer matrices. The retrieved value of $\chi_{\mathrm{m}}^{(2)}$ is in excellent agreement with that predicted by an analytical effective medium theory model. (C) 2010 American Institute of Physics. [doi:10.1063/1.3460919]
\end{abstract}

Metamaterials (MMs) are subwavelength, circuit-like elements whose electromagnetic response can be engineered by design. Near resonance, many MM elements, such as split ring resonators (SRRs), exhibit a significant concentration of the electric field within the capacitive regions, potentially enhancing the nonlinear properties of materials integrated into those regions. ${ }^{1}$ Many nonlinear phenomena such as wave-mixing, ${ }^{2}$ bistability, ${ }^{3}$ and parametric generation ${ }^{4}$ have been predicted in such MMs but implementations are still scarce ${ }^{5,6}$ At radio or microwave frequencies, the nonlinear material can be substituted by nonlinear electronic components. For instance, introducing a varactor into the gap of a SRR yields a MM element with nonlinear magnetizability, which, combined with like SRRs, produces a nonlinear magnetic MM. ${ }^{7,8}$

To date, experimental studies of nonlinear MMs remain mostly qualitative. However, for applications, it is essential to characterize their nonlinearity precisely. Specifically, we want to characterize nonlinear MMs in terms of a series expansion of their nonlinear susceptibility, as a host of well known nonlinear phenomena connect to specific terms of this expansion and it allows direct comparison with conventional materials.

Recently, Larouche and Smith have presented a retrieval method which uses the harmonics generated by a slab of a nonlinear medium to retrieve its effective nonlinear susceptibilities. ${ }^{9}$ Here, we apply the method to retrieve the quadratic nonlinear magnetic susceptibility $\chi_{\mathrm{m}}^{(2)}$ from quantitative measurements of the second harmonic generated by a varactor-loaded SRR (VLSRR) MM. The retrieved nonlinear susceptibility is then compared with the predictions of an analytical theoretical model proposed by Poutrina et al. ${ }^{10}$

To study second harmonic generation (SHG), we reuse the VLSRR MM previously used to study the powerdependant resonance frequency shift, ${ }^{11}$ which is related to the cubic nonlinear susceptibility. Each unit cell of this MM consists of a $17 \mu \mathrm{m}$ thick copper ring on a $0.2 \mathrm{~mm}$ thick FR4 PCB substrate. The ring has an internal radius of $4 \mathrm{~mm}$, is $0.5 \mathrm{~mm}$ wide, and possesses a $1 \mathrm{~mm}$ gap. The gap is loaded with a Skyworks SMV1231 varactor, whose capacitance varies according to

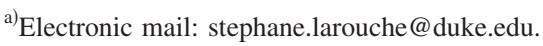

$$
C\left(V_{D}\right)=C_{0}\left(1-V_{D} / V_{p}\right)^{M},
$$

where $V_{D}$ is the bias voltage, $C_{0}=2.4 \mathrm{pF}$ is the zero bias capacitance, $V_{P}=1.5 \mathrm{~V}$ is the intrinsic potential, and $M$ $=0.8$ is the gradient coefficient. ${ }^{12}$ The VLSRRs are arranged periodically to form a slab of $3 \times 15$ cubic unit cells, $10 \mathrm{~mm}$ on a side. The slab is one unit cell in length in the direction of propagation. The unit cells are much smaller than the wavelengths of both the pump and harmonic; the effects of spatial dispersion ${ }^{13}$ can therefore be ignored.

The SHG experiment is performed in a transmission line apparatus similar to that used in Ref. 11 but optimized to maximize transmission at the pump and harmonic frequencies. As shown in Fig. 1, 49\% of the energy of the electromagnetic field is concentrated between the metal plates, where the polarization is nearly transverse electromagnetic. A $10.0 \mathrm{dBm}$ microwave signal of variable frequency $f$ is generated by an Agilent E8267D PSG vector signal generator. Harmonics originating from the signal generator are eliminated using a Mini Circuits VLF-800+ filter. Both the linear transmission and the second harmonic are measured using an Agilent E4440A PSA spectrum analyzer.

The linear transmission of the slab (not shown here) is fitted to determine the permittivity and the permeability of the MM. The former is fitted using a constant value $\epsilon_{\text {slab }}(\omega)=1.63 \epsilon_{0}$, where $\epsilon_{0}$ is the permittivity of vacuum. The latter is fitted using a Lorentz oscillator

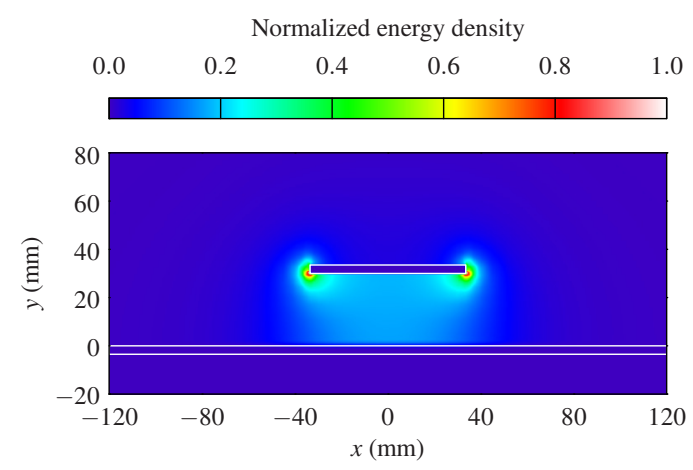

FIG. 1. (Color online) Time-average magnetic energy density in a crosssection of the transmission line determined by simulation using CST MICROWAVE STUDIO. White boxes represent the metal plates. 


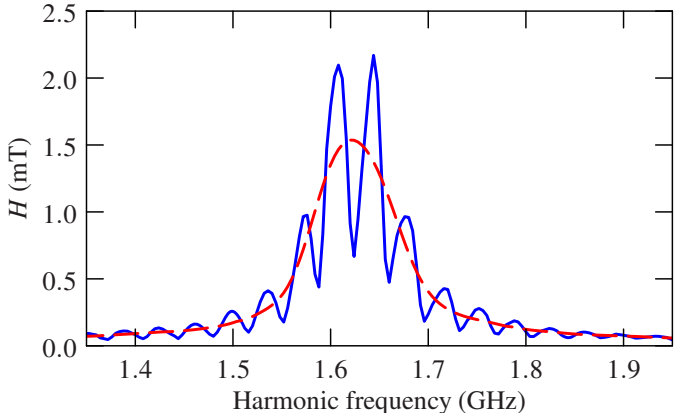

FIG. 2. (Color online) Raw (continuous blue line) and cleaned (dashed red line) transmitted second harmonic.

$$
\mu_{\text {slab }}(\omega)=\mu_{0}\left(1+\frac{F \omega^{2}}{\omega_{0}^{2}-\mathrm{i} \gamma \omega-\omega^{2}}\right)
$$

where $\omega=2 \pi f$ is the angular frequency, $\mu_{0}$ is the permeability of vacuum, $F=0.142$ is the strength of the oscillator, $\omega_{0}=2 \pi \times 812 \mathrm{MHz}$ is the angular resonance frequency, and $\gamma=2 \pi \times 36 \mathrm{MHz}$ is the damping coefficient.

Figure 2 shows the measured second harmonic spectrum. It exhibits oscillations caused by the interference between the transmitted second harmonic and the reflection of the reflected second harmonic by the VLF-800+ filter (as verified by varying the length of the input cable). These oscillations are removed by Fourier analysis and the cleaned second harmonic is superimposed in Fig. 2.

A transfer matrix approach to compute the harmonic generation from a slab of nonlinear material was proposed by Bethune. ${ }^{14}$ It is based on the nondepleted pump approximation, which assumes that nonlinear processes do not significantly alter the distribution of the electromagnetic field at the pump frequency. The approach consists of (1) using a linear transfer matrix approach to calculate the distribution of the electromagnetic field at the pump frequency; (2) calculating the nonlinear polarization which, in turn, generates an electromagnetic field at the harmonic frequencies; and (3) propagating the harmonics to the input and output media using a linear transfer matrix approach. Larouche and Smith have adapted this procedure to determine the nonlinear susceptibility from the harmonic amplitudes. ${ }^{9}$ Electric nonlinearity was studied, but the development can be applied to magnetic nonlinearity by interchanging electric and magnetic fields, polarization and magnetization, and permittivity and permeability in all the final expressions.

For normal incidence and isotropic materials, the magnetic field at any position in the system can be represented by a vector

$$
\vec{H}=\left[\begin{array}{l}
H^{+} \\
H^{-}
\end{array}\right],
$$

consisting of the complex amplitudes of the two contrapropagating solutions to the wave equation. The transfer matrix of a slab of material sandwiched between two semiinfinite media is

$$
M=\left[\begin{array}{ll}
M_{11} & M_{12} \\
M_{21} & M_{22}
\end{array}\right]=M_{\text {slab } \rightarrow \text { out }} \Phi_{\text {slab }} M_{\text {in } \rightarrow \text { slab }},
$$

where the subscripts in, slab, and out refer to the input medium, the nonlinear slab, and the output medium, respectively, while

$$
M_{i \rightarrow j}=\frac{1}{t_{i \rightarrow j}}\left[\begin{array}{cc}
1 & r_{i \rightarrow j} \\
r_{i \rightarrow j} & 1
\end{array}\right] \quad \text { and } \quad \Phi_{\text {slab }}=\left[\begin{array}{cc}
\phi_{\text {slab }} & 0 \\
0 & \phi_{\text {slab }}^{-1}
\end{array}\right]
$$

are the transfer matrix of the interface between medium $i$ and $j$, and the phase transfer matrix of the slab, respectively. In the previous equation, $\phi_{\text {slab }}=\exp \left(\mathrm{i} N_{\text {slab }} k d_{\text {slab }}\right)$ is the phase shift in the slab, $t_{i \rightarrow j}=2 z_{j} /\left(z_{i}+z_{j}\right)$ and $r_{i \rightarrow j}=\left(z_{j}-z_{i}\right) /\left(z_{i}+z_{j}\right)$ are the transmission and reflection coefficients of the interface, respectively, $z_{i}$ and $N_{i}$ are the impedance and refractive index of medium $i$, respectively, $d_{\text {slab }}$ is the thickness of the slab, $k=\omega / c$ is the free-space wavenumber, and $c$ is the speed of light in vacuum. The amplitudes of the two contrapropagating waves in the slab are

$$
\vec{H}_{\text {slab }}=M_{\text {in } \rightarrow \text { slab }}\left[\begin{array}{l}
1 \\
r
\end{array}\right] H_{\text {in }}^{+},
$$

where $r=-M_{21} / M_{22}$ is the reflection coefficient of the composite system and $H_{\text {in }}^{+}$is the applied magnetic field.

If the slab possesses a quadratic nonlinear magnetic susceptibility $\chi_{\mathrm{m}}^{(2)}$, the magnetic field generates a nonlinear magnetization

$$
\vec{M}_{\text {slab }}^{(2)}=\vec{M}_{\text {slab }}^{\left(2,2 N_{\text {slab }} k\right)}+\vec{M}_{\text {slab }}^{\left(2,0 N_{\text {slab }} k\right)},
$$

where

$$
\begin{aligned}
& \vec{M}_{\text {slab }}^{\left(2,2 N_{\text {slab }} k\right)}=\frac{1}{2} \mu_{0} \chi_{\mathrm{m}}^{(2)}\left[\begin{array}{l}
\left(H_{\text {slab }}^{+}\right)^{2} \exp \left(+\mathrm{i} 2 N_{\text {slab }} k z\right) \\
\left(H_{\text {slab }}^{-}\right)^{2} \exp \left(-\mathrm{i} 2 N_{\text {slab }} k z\right)
\end{array}\right], \\
& \vec{M}_{\text {slab }}^{\left(2,0 N_{\text {slab }} k\right)}=\frac{1}{2} \mu_{0} \chi_{\mathrm{m}}^{(2)}\left[\begin{array}{l}
H_{\text {slab }}^{+} H_{\text {slab }}^{-} \\
H_{\text {slab }}^{+} H_{\text {slab }}^{-}
\end{array}\right] .
\end{aligned}
$$

This magnetization, in turn, couples to a magnetic field at the second harmonic frequency

$$
\vec{H}_{\mathrm{s}}=\frac{\vec{M}_{\text {slab }}^{\left(2,2 N_{\text {slab }} k\right)}}{\mu_{\mathrm{s}}^{\left(2 N_{\text {slab }} k\right)}-\mu_{\text {slab }}(2 \omega)}+\frac{\vec{M}_{\text {slab }}^{\left(2,0 N_{\text {slab }} k\right)}}{\mu_{\mathrm{s}}^{\left(0 N_{\text {slab }} k\right)}-\mu_{\text {slab }}(2 \omega)},
$$

where $\mu_{\mathrm{s}}^{(K)}=K^{2} / 4 \omega^{2} \epsilon_{\text {slab }}(2 \omega)$.

Finally, the magnetic field propagates to the input and output media. The reflected $H_{\mathrm{in}}^{(2 \omega),-}$ and transmitted $H_{\text {out }}^{(2 \omega),+}$ second harmonics are

$$
\left[\begin{array}{l}
H_{\text {out }}^{(2 \omega)+} \\
H_{\text {in }}^{(2 \omega),-}
\end{array}\right]=\left[\begin{array}{ll}
R_{11} & -L_{12} \\
R_{21} & -L_{22}
\end{array}\right]^{-1} \vec{S}_{\text {slab }}
$$

where $R=\Phi_{\text {slab }}^{-1} M_{\text {slab } \rightarrow \text { out }}^{-1}, L=M_{\text {in } \rightarrow \text { slab }}$, and

$$
\begin{aligned}
\vec{S}_{\text {slab }}= & \left(\Phi_{\text {slab }}^{-1} M_{\mathrm{s} \rightarrow \text { slab }}^{\left(2 N_{2} k\right)} \Phi_{\mathrm{s}}^{\left(2 N_{2} k\right)}-M_{\mathrm{s} \rightarrow \text { lab }}^{\left(2 N_{2} k\right)}\right) \vec{H}_{\mathrm{s}}^{\left(2 N_{2} k\right)} \\
& +\left(\Phi_{\text {slab }}^{-1} M_{\mathrm{s} \rightarrow \text { slab }}^{\left(0 N_{2} k\right)} \Phi_{\mathrm{s}}^{\left(0 N_{2} k\right)}-M_{\mathrm{s} \rightarrow \text { slab }}^{\left(0 N_{2} k\right)}\right) \vec{H}_{\mathrm{s}}^{\left(0 N_{2} k\right)}
\end{aligned}
$$

is called the source term.

To retrieve $\chi_{m}^{(2)}$, one can compare the measured values of the harmonics with those calculated supposing that $\chi_{\mathrm{m}}^{(2)}=1$; specifically,

$$
\chi_{\mathrm{m}}^{(2)}=\left[\begin{array}{c}
H_{\mathrm{out}}^{(2 \omega),+} \\
H_{\mathrm{in}}^{(2 \omega),-}
\end{array}\right] /\left[\begin{array}{c}
H_{\mathrm{out}}^{(2 \omega),+} \\
H_{\mathrm{in}}^{(2 \omega),-}
\end{array}\right]_{\chi_{\mathrm{m}}^{(2)}=1} .
$$

Note that this equation gives two redundant values of $\chi_{\mathrm{m}}^{(2)}$. In our work, we only measured the transmitted second harmonic. Also note that even though the retrieval method was 


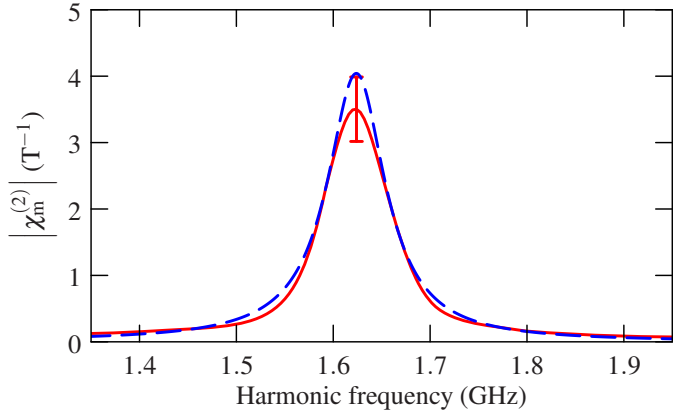

FIG. 3. (Color online) Nonlinear susceptibility determined from experimental results (continuous red line) and comparison with theoretical prediction (dashed blue line).

developed for an isotropic material, it can be used in the present experiment since only one element of the permittivity, permeability, and nonlinear susceptibility tensors are probed.

To determine $\chi_{\mathrm{m}}^{(2)}$ experimentally, the power incident on the sample and that arriving at the spectrum analyzer must be evaluated as accurately as possible. First, we must account for losses. In accordance with measurements done on the whole system and single system elements, we estimate that the pump signal loses $2.5 \pm 0.5 \mathrm{~dB}$ before reaching the sample while the transmitted signal loses $1.0 \pm 0.2 \mathrm{~dB}$ at the pump frequency and $1.3 \pm 0.2 \mathrm{~dB}$ at the second harmonic frequency before reaching the spectrum analyzer. Second, as previously mentioned, only $49 \%$ of the applied power is actually between the plates of the transmission line where the sample is introduced. We assume that the introduction of the slab does not affect significantly this fraction. The magnetic field incident on the slab of MM is $H_{\text {in }}^{+}=\sqrt{2 P / z_{0} S}$ where $P$ is the corrected power, $z_{0}=377 \Omega$ is the impedance of vacuum, and $S=18 \mathrm{~cm}^{2}$ is the cross section of the waveguide.

The retrieved $\chi_{\mathrm{m}}^{(2)}$ can be compared with an analytical expression recently presented by Poutrina et al. ${ }^{10}$ The expression, which results from a perturbative solution of the nonlinear oscillator model describing the effective RLC circuit of the SRR, is

$$
\chi_{\mathrm{m}}^{(2)}(2 \omega, \omega, \omega)=-\mathrm{i} a \frac{2 \omega_{0}^{4} \omega^{3} \mu_{0}^{2} A F}{D^{2}(\omega) D(2 \omega)},
$$

where $A$ is the area of the VLSRR, $a=-M / 2 V_{p}$ is related to the second order coefficient in the Taylor expansion of the induced bias voltage $V_{D}$ in terms of the oscillating charge, and $D(\omega)=\omega_{0}^{2}-\mathrm{i} \gamma \omega-\omega^{2}$ is the denominator of the Lorentz oscillator at angular frequency $\omega$.

Figure 3 shows the amplitude of $\chi_{\mathrm{m}}^{(2)}$ extracted from the experimental measurements and that predicted by the analytical model. Only the amplitude is shown since the spectrum analyzer does not provide phase information. We find a good agreement between the value of $\chi_{m}^{(2)}$ predicted by Eq.
(14) and that determined experimentally. This agreement, which supports the validity of the theoretical approach, is remarkable considering that, while we have fit $\mu(\omega)$ to the linear transmission spectrum of the MM slab and used these values in Eq. (14), there is absolutely no fit of the parameters controlling the nonlinearity. However, some caution is necessary. We ignore the effect of $\chi_{\mathrm{m}}^{(4)}, \chi_{\mathrm{m}}^{(6)}, \ldots$ on the SHG in the retrieval, effectively making the retrieved $\chi_{\mathrm{m}}^{(2)}$ power dependent if higher-order terms are significant. It is not excluded that the almost perfect agreement is an effect of this approximation and those involved in the perturbative approach that led to Eq. (14).

The value of $\chi_{\mathrm{m}}^{(2)}$ for the VLSRR MM is many orders of magnitude higher than that observed in naturally occurring nonlinear materials. However, because of the small thickness of the slab, only a very small fraction of the applied field is actually converted into harmonics, validating the nondepleted pump approximation. This large value of $\chi_{\mathrm{m}}^{(2)}$ arises from the large nonlinearity of the varactor. It is further enhanced by the concentration of the electric field in the gap of the SRR, which itself relates to the operation of the SRR based MM near its resonance frequency. The gigantic value of $\chi_{\mathrm{m}}^{(2)}$ confirms earlier qualitative predictions.

This work was supported by the Air Force Office of Scientific Research (Contract No. FA9550-09-1-0562) and by the U.S. Army (Contract No. W31P4Q-09-C-0648). We thank Daniel Gauthier and Gao Zheng for access to equipment and helpful discussions. S. L. thanks the Natural Sciences and Engineering Research Council of Canada for his postdoctoral fellowship.

${ }^{1}$ J. B. Pendry, A. J. Holden, D. J. Robbins, and W. J. Stewart, IEEE Trans. Microwave Theory Tech. 47, 2075 (1999).

${ }^{2}$ A. K. Popov, S. A. Myslivets, T. F. George, and V. M. Shalaev, Opt. Lett. 32, 3044 (2007).

${ }^{3}$ S. O'Brien, D. McPeake, S. A. Ramakrishna, and J. B. Pendry, Phys. Rev. B 69, 241101(R) (2004).

${ }^{4}$ E. Poutrina, S. Larouche, and D. R. Smith, Opt. Commun. 283, 1640 (2010).

${ }^{5}$ M. W. Klein, C. Enkrich, M. Wegener, and S. Linden, Science 313, 502 (2006).

${ }^{6}$ E. Kim, F. Wang, W. Wu, Z. Yu, and Y. R. Shen, Phys. Rev. B 78, 113102 (2008).

${ }^{7}$ I. V. Shadrivov, S. K. Morrison, and Y. S. Kivshar, Opt. Express 14, 9344 (2006).

${ }^{8}$ B. Wang, J. Zhou, T. Koschny, and C. M. Soukoulis, Opt. Express 16, 16058 (2008).

${ }^{9}$ S. Larouche and D. R. Smith, Opt. Commun. 283, 1621 (2010).

${ }^{10}$ E. Poutrina, D. Huang, and D. R. Smith, arXiv:1004.2784v1 (unpublished).

${ }^{11}$ D. Huang, E. Poutrina, and D. R. Smith, Appl. Phys. Lett. 96, 104104 (2010).

${ }^{12}$ Skyworks, "Varactor SPICE models for RF VCO applications," http:// www.skyworksinc.com/uploads/documents/200315B.pdf.

${ }^{13}$ C. R. Simovski and S. A. Tretyakov, Phys. Rev. B 75, 195111 (2007).

${ }^{14}$ D. S. Bethune, J. Opt. Soc. Am. B 6, 910 (1989). 